\title{
Supporting Information for Distonic Isomers and Tautomers of Adenine Cation Radical in the Gas Phase and Aqueous Solution
}

Xiaohong Chen $^{\dagger}$, Erik A. Syrstad ${ }^{\dagger}$, Minh Tho Nguyen ${ }^{*}$, Pascal Gerbaux ${ }^{\mathbb{I I} *}$, and František Tureček $^{\dagger *}$

'Department of Chemistry, Bagley Hall, Box 351700, University of Washington, Seattle, WA 98195-1700, USA

Department of Chemistry, University of Leuven, Leuven, Belgium

"Department of Organic Chemistry, University of Mons-Hainaut, Mons, Belgium

Tables S1-S22 with optimized structures of adenine, adenine tautomers, protonated adenine tautomers, triplet states of cations, and cation-radicals in Cartesian coordinate format. Figures S1-S4 with optimized structures and molecular orbitals.

Table S1. Adenine (1), B3LYP/6-31+G(d,p) optimization

\begin{tabular}{|c|c|c|c|c|c|}
\hline \multirow{2}{*}{$\begin{array}{l}\text { Center } \\
\text { Number }\end{array}$} & \multirow{2}{*}{$\begin{array}{l}\text { Atomic } \\
\text { Number }\end{array}$} & \multirow{2}{*}{$\begin{array}{l}\text { Atomic } \\
\text { Type }\end{array}$} & \multicolumn{3}{|c|}{ Coordinates (Angstroms) } \\
\hline & & & $\mathrm{X}$ & $\mathrm{Y}$ & $\mathrm{z}$ \\
\hline 1 & 7 & 0 & 1.948435 & .527344 & .000457 \\
\hline 2 & 6 & 0 & 1.295531 & 1.702577 & .000377 \\
\hline 3 & 7 & 0 & -.023502 & 1.923169 & -.000082 \\
\hline 4 & 6 & 0 & -.711267 & .773822 & -.000291 \\
\hline 5 & 6 & 0 & -.179693 & -.520777 & -.000440 \\
\hline 6 & 6 & 0 & 1.229239 & -.609940 & -.000415 \\
\hline 7 & 7 & 0 & -1.182312 & -1.477396 & .000096 \\
\hline 8 & 6 & 0 & -2.292108 & -.777274 & .000347 \\
\hline 9 & 7 & 0 & -2.076865 & .586987 & .000070 \\
\hline 10 & 7 & 0 & 1.881531 & -1.796815 & -.004024 \\
\hline 11 & 1 & 0 & 1.927829 & 2.586659 & .000956 \\
\hline 12 & 1 & 0 & -3.290610 & -1.192716 & .000767 \\
\hline 13 & 1 & 0 & -2.773933 & 1.317449 & .000173 \\
\hline 14 & 1 & 0 & 2.889056 & -1.802663 & .012291 \\
\hline 15 & 1 & 0 & 1.366437 & -2.662207 & .012731 \\
\hline Rotatic & consta & ( GHZ & 3678824 & 1.5631110 & .941561 \\
\hline
\end{tabular}


Table S2. Adenine cation-radical (1+•), B3LYP/6-31+G(d,p) optimization.

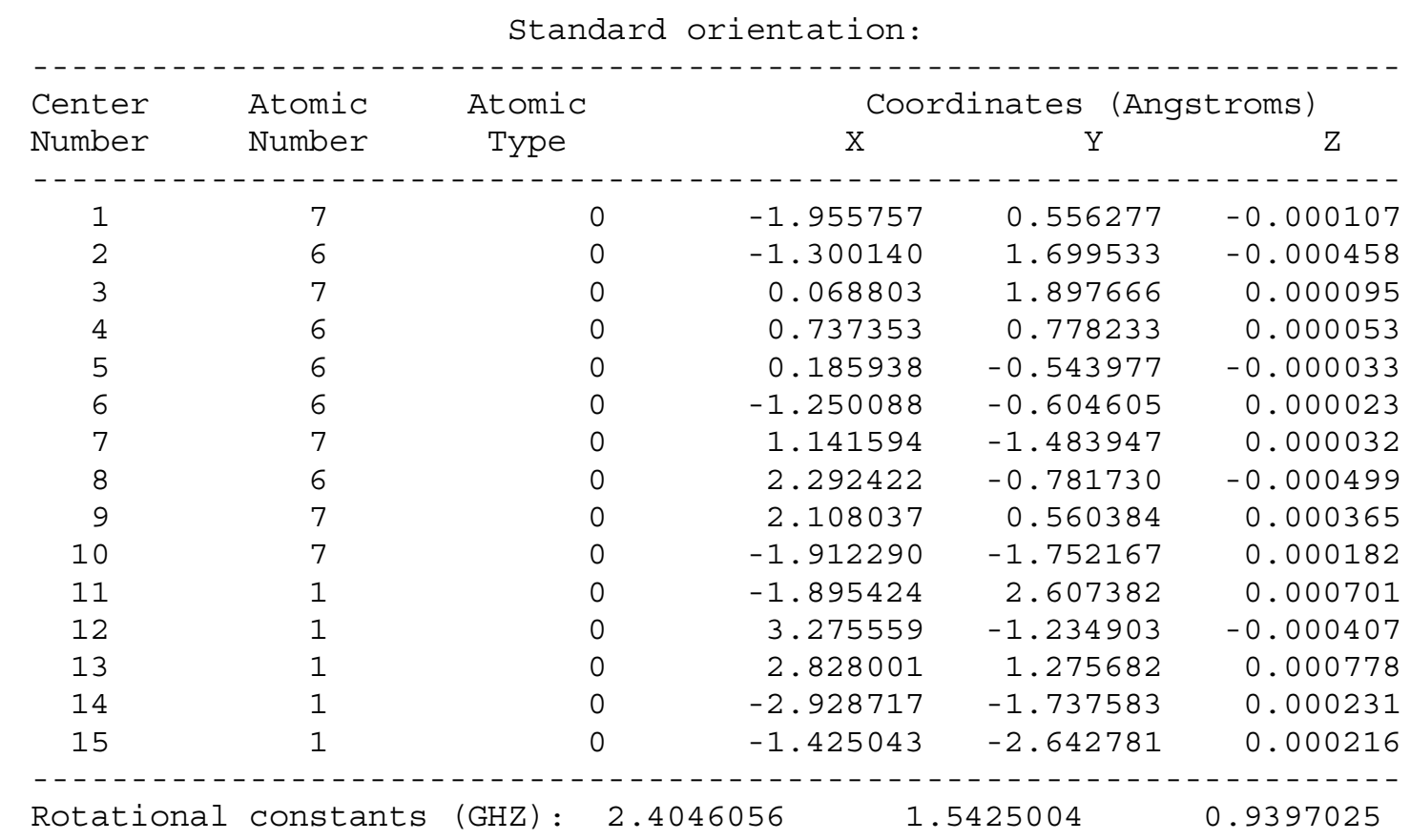

Table S3. Adenine protonated at N-1 $\left(\mathbf{2}^{+}\right)$. B3LYP/6-311++G(d,p) optimization. $\mathrm{Z}$-Matrix orientation:

\begin{tabular}{|c|c|c|c|c|c|}
\hline \multirow{2}{*}{$\begin{array}{l}\text { Center } \\
\text { Number }\end{array}$} & \multirow{2}{*}{$\begin{array}{l}\text { Atomic } \\
\text { Number }\end{array}$} & \multirow{2}{*}{$\begin{array}{c}\text { Atomic } \\
\text { Type }\end{array}$} & \multicolumn{3}{|c|}{ Coordinates (Angstroms) } \\
\hline & & & $\mathrm{X}$ & $\mathrm{Y}$ & Z \\
\hline & & & ------- & $-\ldots$ & - - - - - - \\
\hline 1 & 7 & 0 & .000000 & .000000 & .000000 \\
\hline 2 & 6 & 0 & .000000 & .000000 & 1.383555 \\
\hline 3 & 7 & 0 & 1.071316 & .000000 & 2.111814 \\
\hline 4 & 6 & 0 & 2.212272 & .000001 & 1.393163 \\
\hline 5 & 6 & 0 & 2.324101 & .000003 & -.002024 \\
\hline 6 & 6 & 0 & 1.141794 & .000002 & -.757073 \\
\hline 7 & 7 & 0 & 3.634565 & .000005 & -.401667 \\
\hline 8 & 6 & 0 & 4.310172 & .000004 & .720291 \\
\hline 9 & 7 & 0 & 3.498186 & .000002 & 1.838787 \\
\hline 10 & 7 & 0 & 1.104432 & .000003 & -2.086369 \\
\hline 11 & 1 & 0 & -.980680 & -.000001 & 1.844361 \\
\hline 12 & 1 & 0 & 5.387272 & .000005 & .800679 \\
\hline 13 & 1 & 0 & 3.793780 & .000001 & 2.807233 \\
\hline 14 & 1 & 0 & .249310 & .000002 & -2.621832 \\
\hline 15 & 1 & 0 & 1.980793 & .000004 & -2.591891 \\
\hline 16 & 1 & 0 & -.902920 & -.000001 & -.461553 \\
\hline atı & onsta & & 1.564 & .93 & 194 \\
\hline
\end{tabular}


Table S4. Adenine protonated at C-2 . B3LYP/6-311++G(d,p) optimization.

Standard orientation:

\begin{tabular}{|c|c|c|c|c|c|}
\hline \multirow{2}{*}{$\begin{array}{l}\text { Center } \\
\text { Number }\end{array}$} & \multirow{2}{*}{$\begin{array}{l}\text { Atomic } \\
\text { Number }\end{array}$} & \multirow{2}{*}{$\begin{array}{c}\text { Atomic } \\
\text { Type }\end{array}$} & \multicolumn{3}{|c|}{ Coordinates (Angstroms) } \\
\hline & & & $\mathrm{X}$ & $\mathrm{Y}$ & $\mathrm{Z}$ \\
\hline \multicolumn{6}{|c|}{ - - - - - - - - - - - - - - - - - - - - - - - - - - - - - - - - - - - - - - - - - - - - - - - - - - - - - - - - - - } \\
\hline 1 & 7 & 0 & -1.683197 & -1.064340 & .000000 \\
\hline 2 & 6 & 0 & -.670901 & -2.048738 & .000000 \\
\hline 3 & 7 & 0 & .730462 & -1.751541 & .000000 \\
\hline 4 & 6 & 0 & .964806 & -.495111 & .000000 \\
\hline 5 & 6 & 0 & .000000 & .581376 & .000000 \\
\hline 6 & 6 & 0 & -1.399214 & .217794 & .000000 \\
\hline 7 & 7 & 0 & .596225 & 1.765859 & .000000 \\
\hline 8 & 6 & 0 & 1.919753 & 1.484649 & .000000 \\
\hline 9 & 7 & 0 & 2.196424 & .176806 & .000000 \\
\hline 10 & 7 & 0 & -2.350263 & 1.160368 & .000000 \\
\hline 11 & 1 & 0 & -.841093 & -2.733020 & .853642 \\
\hline 12 & 1 & 0 & -.841093 & -2.733020 & -.853642 \\
\hline 13 & 1 & 0 & 2.690047 & 2.244778 & .000000 \\
\hline 14 & 1 & 0 & 3.113840 & -.256004 & .000000 \\
\hline 15 & 1 & 0 & -3.322081 & .886822 & .000000 \\
\hline 16 & 1 & 0 & -2.113847 & 2.140552 & .000000 \\
\hline \multicolumn{6}{|c|}{ - - - - - - - - - - - - - - - - - - - - - - - - - - - - - - - - - - - - - - - - - - - - - - - - - - - - - - - - - - - - - - - - } \\
\hline Rotati & constar & $(\mathrm{GHZ}):$ & 5762 & 5396182 & .9235803 \\
\hline
\end{tabular}

Table S5. Adenine protonated at N-3 $\left(3^{+}\right)$. B3LYP/6-311++G(d,p) optimization.

Z-Matrix orientation:

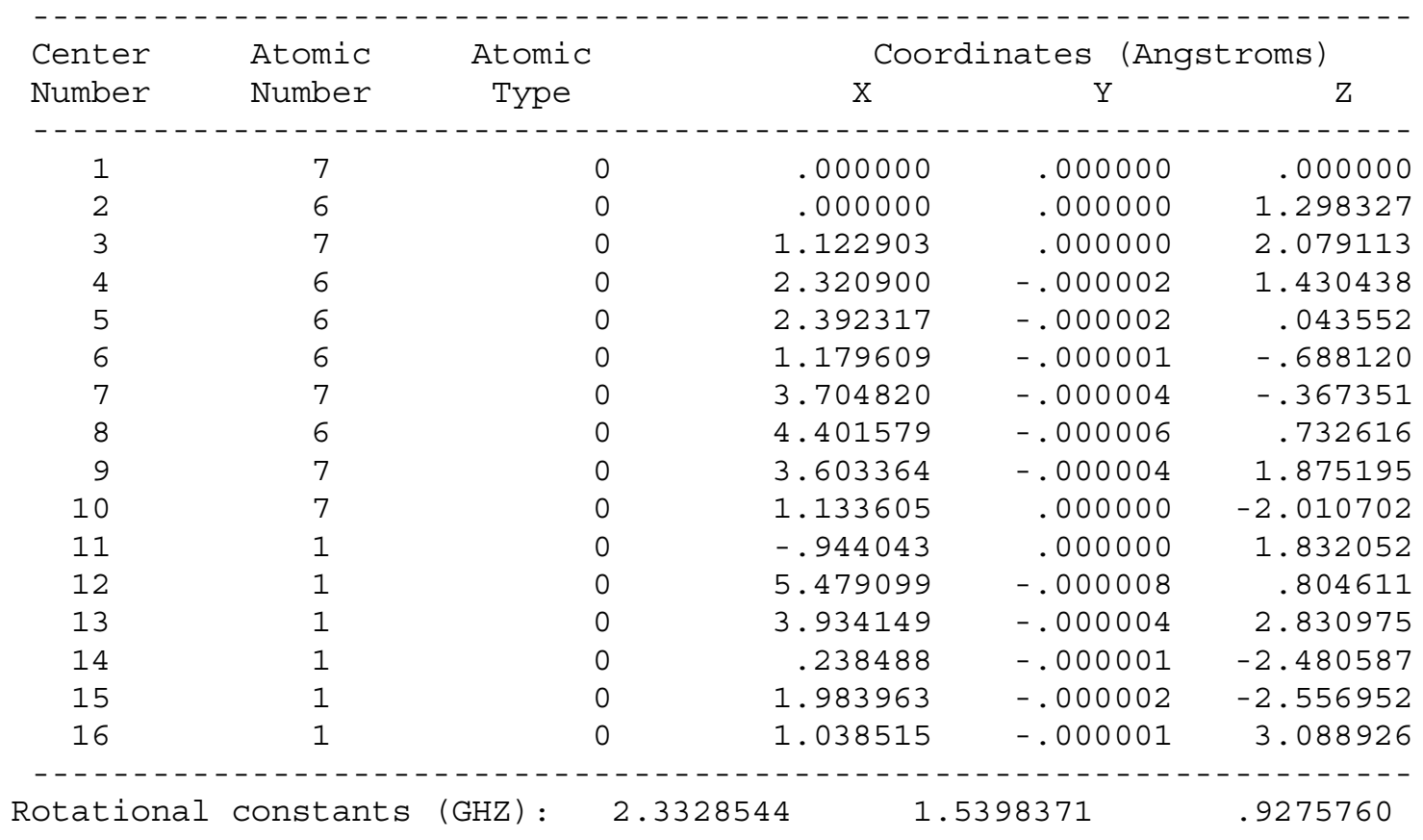


Table S6. Adenine protonated at C-4 (3+) . B3LYP/6-311++G(d,p) optimization.

standard orientation:

\begin{tabular}{|c|c|c|c|c|c|}
\hline \multirow{2}{*}{$\begin{array}{l}\text { Center } \\
\text { Number }\end{array}$} & \multirow{2}{*}{$\begin{array}{l}\text { Atomic } \\
\text { Number }\end{array}$} & \multirow{2}{*}{$\begin{array}{c}\text { Atomic } \\
\text { Type }\end{array}$} & \multicolumn{3}{|c|}{ Coordinates (Angstroms) } \\
\hline & & & $\mathrm{X}$ & $\mathrm{Y}$ & Z \\
\hline \multicolumn{6}{|c|}{ - - - - - - - - - - - - - - - - - - - - - - - - - - - - - - - - - - - - - - - - - - - - - - - - - - - - - - - - - - } \\
\hline 1 & 7 & 0 & 1.857149 & .685671 & -.184494 \\
\hline 2 & 6 & 0 & 1.030821 & 1.792602 & -.272421 \\
\hline 3 & 7 & 0 & -.233334 & 1.902806 & -.017755 \\
\hline 4 & 6 & 0 & -.803115 & .686948 & .482276 \\
\hline 5 & 6 & 0 & -.107525 & -.580647 & .137713 \\
\hline 6 & 6 & 0 & 1.357879 & -.497244 & .069293 \\
\hline 7 & 7 & 0 & -.906958 & -1.527157 & -.274737 \\
\hline 8 & 6 & 0 & -2.157713 & -.948668 & -.272815 \\
\hline 9 & 7 & 0 & -2.163726 & .311753 & .072760 \\
\hline 10 & 7 & 0 & 2.105184 & -1.597139 & .153975 \\
\hline 11 & 1 & 0 & 1.537964 & 2.695658 & -.599641 \\
\hline 12 & 1 & 0 & -3.052914 & -1.500143 & -.534987 \\
\hline 13 & 1 & 0 & -2.978826 & .913916 & .143340 \\
\hline 14 & 1 & 0 & 3.103393 & -1.525632 & .004412 \\
\hline 15 & 1 & 0 & 1.693309 & -2.508750 & .286320 \\
\hline 16 & 1 & 0 & -.833206 & .775475 & 1.588045 \\
\hline \multicolumn{6}{|c|}{ - - - - - - - - - - - - - - - - - - - - - - - - - - - - - - - - - - - - - - - - - - - - - - - - - - - - - - - - - - - - - - } \\
\hline Rotati & constar & (GHZ) : & 381 & 5266329 & .9542445 \\
\hline
\end{tabular}

Table S7. Adenine protonated at C-5. B3LYP/6-311++G(d,p) optimization.

Standard orientation:

\begin{tabular}{|c|c|c|c|c|c|}
\hline \multirow{2}{*}{$\begin{array}{l}\text { Center } \\
\text { Number }\end{array}$} & \multirow{2}{*}{$\begin{array}{l}\text { Atomic } \\
\text { Number }\end{array}$} & \multirow{2}{*}{$\begin{array}{l}\text { Atomic } \\
\text { Type }\end{array}$} & \multicolumn{3}{|c|}{ Coordinates (Angstroms) } \\
\hline & & & $\mathrm{X}$ & $\mathrm{Y}$ & Z \\
\hline \multicolumn{6}{|c|}{ - - - - - - - - - - - - - - - - - - - - - - - - - - - - - - - - - - - - - - - - - - - - - - - - - - - } \\
\hline 1 & 7 & 0 & -1.974416 & .427755 & -.089575 \\
\hline 2 & 6 & 0 & -1.395963 & 1.625835 & .004547 \\
\hline 3 & 7 & 0 & -.072071 & 1.922755 & -.016863 \\
\hline 4 & 6 & 0 & .683518 & .867637 & .097909 \\
\hline 5 & 6 & 0 & .203700 & -.492867 & .504094 \\
\hline 6 & 6 & 0 & -1.205236 & -.658714 & .008174 \\
\hline 7 & 7 & 0 & 1.267627 & -1.426782 & .167839 \\
\hline 8 & 6 & 0 & 2.255947 & -.691190 & -.174152 \\
\hline 9 & 7 & 0 & 1.979026 & .697925 & -.205915 \\
\hline 10 & 7 & 0 & -1.683447 & -1.855016 & -.231676 \\
\hline 11 & 1 & 0 & -2.068561 & 2.477810 & .018013 \\
\hline 12 & 1 & 0 & 3.242195 & -1.051511 & -.438461 \\
\hline 13 & 1 & 0 & 2.605805 & 1.429141 & -.525221 \\
\hline 14 & 1 & 0 & -2.660709 & -1.962052 & -.483655 \\
\hline 15 & 1 & 0 & -1.087578 & -2.673344 & -.187056 \\
\hline 16 & 1 & 0 & .100019 & -.490719 & 1.606281 \\
\hline \multicolumn{6}{|c|}{ - - - - - - - - - - - - - - - - - - - - - - - - - - - - - - - - - - - - - - - - - - - - - - - - - - - - - - - - - - - - - - - - } \\
\hline Rotati & constar & (GHZ) : & 93881 & 5862229 & .9665389 \\
\hline
\end{tabular}


Table S8. Adenine protonated at C-6. B3LYP/6-311++G(d,p) optimization. Standard orientation:

\begin{tabular}{|c|c|c|c|c|c|}
\hline \multirow{2}{*}{$\begin{array}{l}\text { Center } \\
\text { Number }\end{array}$} & \multirow{2}{*}{$\begin{array}{l}\text { Atomic } \\
\text { Number }\end{array}$} & \multirow{2}{*}{$\begin{array}{l}\text { Atomic } \\
\text { Type }\end{array}$} & \multicolumn{3}{|c|}{ Coordinates (Angstroms) } \\
\hline & & & $\mathrm{X}$ & $\mathrm{Y}$ & $\mathrm{z}$ \\
\hline 1 & 7 & 0 & 1.921150 & .661243 & .029478 \\
\hline 2 & 6 & 0 & 1.238298 & 1.755980 & -.041918 \\
\hline 3 & 7 & 0 & -.152780 & 1.883918 & -.070078 \\
\hline 4 & 6 & 0 & -.783365 & .767610 & .022411 \\
\hline 5 & 6 & 0 & -.199120 & -.560964 & .057639 \\
\hline 6 & 6 & 0 & 1.258754 & -.596632 & .199655 \\
\hline 7 & 7 & 0 & -1.131948 & -1.501413 & .034714 \\
\hline 8 & 6 & 0 & -2.295173 & -.821924 & -.025797 \\
\hline 9 & 7 & 0 & -2.151109 & .516141 & -.038491 \\
\hline 10 & 7 & 0 & 1.983003 & -1.707832 & -.272151 \\
\hline 11 & 1 & 0 & 1.779542 & 2.693003 & -.109907 \\
\hline 12 & 1 & 0 & -3.264215 & -1.301323 & -.075144 \\
\hline 13 & 1 & 0 & -2.886998 & 1.212086 & -.094847 \\
\hline 14 & 1 & 0 & 2.978523 & -1.648978 & -.091779 \\
\hline 15 & 1 & 0 & 1.591486 & -2.611443 & -.035561 \\
\hline 16 & 1 & 0 & 1.207083 & -.572177 & 1.350992 \\
\hline Rotatic & const & (GHZ) & 341 & 984043 & .9253299 \\
\hline
\end{tabular}

Table S8. Adenine protonated at N-7 (4+). B3LYP/6-311++G(d,p) optimization. $\mathrm{Z}$-Matrix orientation:

\begin{tabular}{|c|c|c|c|c|c|}
\hline \multirow{2}{*}{$\begin{array}{l}\text { Center } \\
\text { Number }\end{array}$} & \multirow{2}{*}{$\begin{array}{l}\text { Atomic } \\
\text { Number }\end{array}$} & \multirow{2}{*}{$\begin{array}{c}\text { Atomic } \\
\text { Type }\end{array}$} & \multicolumn{3}{|c|}{ Coordinates (Angstroms) } \\
\hline & & & $\mathrm{x}$ & $\mathrm{Y}$ & Z \\
\hline 1 & 7 & 0 & .000000 & .000000 & .000000 \\
\hline 2 & 6 & 0 & .000000 & .000000 & 1.334719 \\
\hline 3 & 7 & 0 & 1.062021 & .000000 & 2.150719 \\
\hline 4 & 6 & 0 & 2.193594 & .000001 & 1.474195 \\
\hline 5 & 6 & 0 & 2.343238 & .000001 & .087630 \\
\hline 6 & 6 & 0 & 1.151805 & .000001 & -.682382 \\
\hline 7 & 7 & 0 & 3.718934 & .000004 & -.158870 \\
\hline 8 & 6 & 0 & 4.370836 & .000004 & 1.004666 \\
\hline 9 & 7 & 0 & 3.485265 & .000002 & 2.002649 \\
\hline 10 & 7 & 0 & 1.093890 & .000002 & -2.024963 \\
\hline 11 & 1 & 0 & -.974347 & -.000001 & 1.810504 \\
\hline 12 & 1 & 0 & 5.443670 & .000005 & 1.116763 \\
\hline 13 & 1 & 0 & 3.706432 & .000002 & 2.992442 \\
\hline 14 & 1 & 0 & .179168 & -.000001 & -2.454475 \\
\hline 15 & 1 & 0 & 1.901101 & -.000003 & -2.624666 \\
\hline 16 & 1 & 0 & 4.186118 & .000005 & -1.056209 \\
\hline Rotati & onsta & & 7 & 9729 & .9198038 \\
\hline
\end{tabular}


Table S9. Adenine protonated at C-8 $\left(\mathbf{4}^{+}\right)$. B3LYP/6-311++G(d,p) optimization. Standard orientation:

\begin{tabular}{|c|c|c|c|c|c|}
\hline \multirow{2}{*}{$\begin{array}{l}\text { Center } \\
\text { Number }\end{array}$} & \multirow{2}{*}{$\begin{array}{l}\text { Atomic } \\
\text { Number }\end{array}$} & \multirow{2}{*}{$\begin{array}{l}\text { Atomic } \\
\text { Type }\end{array}$} & \multicolumn{3}{|c|}{ Coordinates (Angstroms) } \\
\hline & & & $\mathrm{X}$ & $\mathrm{Y}$ & Z \\
\hline \multicolumn{6}{|c|}{ 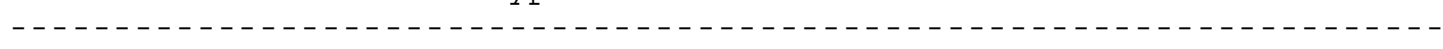 } \\
\hline 1 & 7 & 0 & 1.754832 & -1.075115 & .000000 \\
\hline 2 & 6 & 0 & .780457 & -1.999870 & .000000 \\
\hline 3 & 7 & 0 & -.557176 & -1.847423 & .000000 \\
\hline 4 & 6 & 0 & -.924583 & -.583065 & .000000 \\
\hline 5 & 6 & 0 & .000000 & .562310 & .000000 \\
\hline 6 & 6 & 0 & 1.428320 & .213486 & .000000 \\
\hline 7 & 7 & 0 & -.595363 & 1.697048 & .000000 \\
\hline 8 & 6 & 0 & -2.012774 & 1.417867 & .000000 \\
\hline 9 & 7 & 0 & -2.135720 & -.041890 & .000000 \\
\hline 10 & 7 & 0 & 2.355711 & 1.151274 & .000000 \\
\hline 11 & 1 & 0 & 1.128497 & -3.028344 & .000000 \\
\hline 12 & 1 & 0 & -2.477383 & 1.863955 & .887779 \\
\hline 13 & 1 & 0 & -2.477383 & 1.863955 & -.887779 \\
\hline 14 & 1 & 0 & -3.004550 & -.564581 & .000000 \\
\hline 15 & 1 & 0 & 3.332449 & .879745 & .000000 \\
\hline 16 & 1 & 0 & 2.113867 & 2.133651 & .000000 \\
\hline \multicolumn{6}{|c|}{ - - - - - - - - - - - - - - - - - - - - - - - - - - - - - - - - - - - - - - - - - - - - - - - - - - - - - - - - - - - - } \\
\hline Rotatic & constar & $(\mathrm{GHZ}):$ & 908 & 88249 & .9215458 \\
\hline
\end{tabular}

Table S10. Adenine protonated at N-9. B3LYP/6-311++G(d,p) optimization.

Standard orientation:

\begin{tabular}{|c|c|c|c|c|c|}
\hline \multirow{2}{*}{$\begin{array}{l}\text { Center } \\
\text { Number }\end{array}$} & \multirow{2}{*}{$\begin{array}{l}\text { Atomic } \\
\text { Number }\end{array}$} & \multirow{2}{*}{$\begin{array}{l}\text { Atomic } \\
\text { Type }\end{array}$} & \multicolumn{3}{|c|}{ Coordinates (Angstroms) } \\
\hline & & & $\mathrm{X}$ & $\mathrm{Y}$ & Z \\
\hline \multicolumn{6}{|c|}{ 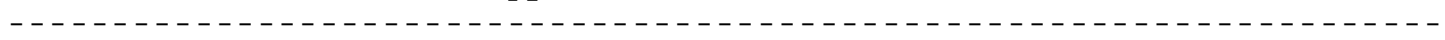 } \\
\hline 1 & 7 & 0 & 1.774961 & -1.021814 & .000000 \\
\hline 2 & 6 & 0 & .870999 & -1.982273 & .000000 \\
\hline 3 & 7 & 0 & -.485215 & -1.835806 & .000000 \\
\hline 4 & 6 & 0 & -.806965 & -.579160 & .000000 \\
\hline 5 & 6 & 0 & .000000 & .538152 & .000000 \\
\hline 6 & 6 & 0 & 1.392295 & .278785 & .000000 \\
\hline 7 & 7 & 0 & -. 737782 & 1.732287 & .000000 \\
\hline 8 & 6 & 0 & -1.968829 & 1.451541 & .000000 \\
\hline 9 & 7 & 0 & -2.209402 & -.060823 & .000000 \\
\hline 10 & 7 & 0 & 2.332432 & 1.222924 & .000000 \\
\hline 11 & 1 & 0 & 1.236511 & -3.002915 & .000000 \\
\hline 12 & 1 & 0 & -2.827817 & 2.107625 & .000000 \\
\hline 13 & 1 & 0 & -2.731902 & -.376151 & .827726 \\
\hline 14 & 1 & 0 & -2.731902 & -.376151 & -.827726 \\
\hline 15 & 1 & 0 & 3.303330 & .943320 & .000000 \\
\hline 16 & 1 & 0 & 2.101817 & 2.204625 & .000000 \\
\hline---------- & - & & -------- & -------- & --------- \\
\hline Rotational & constants & (GHZ): & 17 & 957727 & .9160954 \\
\hline
\end{tabular}


Table S11. Adenine protonated at N-10 (5+). B3LYP/6-311++G(d,p) optimization.

Standard orientation:

\begin{tabular}{|c|c|c|c|c|c|}
\hline \multirow{2}{*}{$\begin{array}{l}\text { Center } \\
\text { Number }\end{array}$} & \multirow{2}{*}{$\begin{array}{l}\text { Atomic } \\
\text { Number }\end{array}$} & \multirow{2}{*}{$\begin{array}{l}\text { Atomic } \\
\text { Type }\end{array}$} & \multicolumn{3}{|c|}{ Coordinates (Angstroms) } \\
\hline & & & $\mathrm{X}$ & $\mathrm{Y}$ & $\mathrm{Z}$ \\
\hline & & & --- & --- & $-\ldots--$ \\
\hline 1 & 7 & 0 & -1.524258 & -1.269258 & .000000 \\
\hline 2 & 6 & 0 & -.463728 & -2.107520 & .000000 \\
\hline 3 & 7 & 0 & .819980 & -1.749946 & .000000 \\
\hline 4 & 6 & 0 & 1.014771 & -.441845 & .000000 \\
\hline 5 & 6 & 0 & .000000 & .549324 & .000000 \\
\hline 6 & 6 & 0 & -1.271576 & .015951 & .000000 \\
\hline 7 & 7 & 0 & .528510 & 1.815083 & .000000 \\
\hline 8 & 6 & 0 & 1.825903 & 1.611408 & .000000 \\
\hline 9 & 7 & 0 & 2.179183 & .280533 & .000000 \\
\hline 10 & 7 & 0 & -2.477629 & .896641 & .000000 \\
\hline 11 & 1 & 0 & -.688130 & -3.166758 & .000000 \\
\hline 12 & 1 & 0 & 2.571486 & 2.393590 & .000000 \\
\hline 13 & 1 & 0 & 3.117457 & -.099052 & .000000 \\
\hline 14 & 1 & 0 & -3.306511 & .288748 & .000000 \\
\hline 15 & 1 & 0 & -2.503515 & 1.504097 & .827627 \\
\hline 16 & 1 & 0 & -2.503515 & 1.504097 & -.827627 \\
\hline & & & ------- & -------- & ------- \\
\hline Rotati & const & (GHZ) & 50391 & 5287438 & .9221771 \\
\hline
\end{tabular}

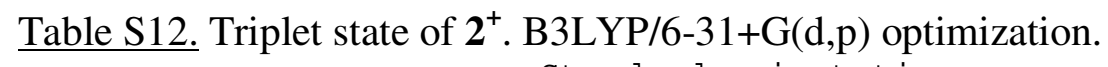

Standard orientation:

\begin{tabular}{|c|c|c|c|c|c|}
\hline \multirow{2}{*}{$\begin{array}{l}\text { Center } \\
\text { Number }\end{array}$} & \multirow{2}{*}{$\begin{array}{l}\text { Atomic } \\
\text { Number }\end{array}$} & \multirow{2}{*}{$\begin{array}{l}\text { Atomic } \\
\text { Type }\end{array}$} & \multicolumn{3}{|c|}{ Coordinates (Angstroms) } \\
\hline & & & $\mathrm{x}$ & $\mathrm{Y}$ & Z \\
\hline 1 & 7 & 0 & 1.898828 & 520732 & -173621 \\
\hline 2 & 6 & 0 & 1.294836 & 1.697286 & .022019 \\
\hline 3 & 7 & 0 & -.022512 & 1.902511 & .130329 \\
\hline 4 & 6 & 0 & -.733951 & .773880 & .051624 \\
\hline 5 & 6 & 0 & -.237756 & -.553887 & -.149994 \\
\hline 6 & 6 & 0 & 1.203753 & -.748072 & -.298385 \\
\hline 7 & 7 & 0 & -1.226856 & -1.438822 & -.182879 \\
\hline 8 & 6 & 0 & -2.360691 & -.702904 & -.016724 \\
\hline 9 & 7 & 0 & -2.097791 & .624107 & .109556 \\
\hline 10 & 7 & 0 & 1.777524 & -1.823197 & .349649 \\
\hline 11 & 1 & 0 & 1.945961 & 2.565299 & .084292 \\
\hline 12 & 1 & 0 & -3.359903 & -1.114232 & .011364 \\
\hline 13 & 1 & 0 & -2.772075 & 1.369890 & .245848 \\
\hline 14 & 1 & 0 & 2.774545 & -1.990765 & .280784 \\
\hline 15 & 1 & 0 & 1.208917 & -2.660125 & .427404 \\
\hline 16 & 1 & 0 & 2.901055 & .534796 & -.332173 \\
\hline Rotatic & const & Z) & 22678 & 5111809 & .9284884 \\
\hline
\end{tabular}


Table S13. Triplet state of $\mathbf{3}^{+}$. B3LYP/6-31+G(d,p) optimization.

Standard orientation:

\begin{tabular}{|c|c|c|c|c|c|}
\hline \multirow{2}{*}{$\begin{array}{l}\text { Center } \\
\text { Number }\end{array}$} & \multirow{2}{*}{$\begin{array}{l}\text { Atomic } \\
\text { Number }\end{array}$} & \multirow{2}{*}{$\begin{array}{l}\text { Atomic } \\
\text { Type }\end{array}$} & \multicolumn{3}{|c|}{ Coordinates (Angstroms) } \\
\hline & & & $\mathrm{X}$ & Y & Z \\
\hline \multicolumn{6}{|c|}{ - - - - - - - - - - - - - - - - - - - - - - - - - - - - - - - - - - - - - - - - - - - - - - - - - - - - - } \\
\hline 1 & 7 & 0 & 1.976148 & .506996 & -.000264 \\
\hline 2 & 6 & 0 & 1.422925 & 1.682628 & -.000329 \\
\hline 3 & 7 & 0 & -.047050 & 1.854371 & .000146 \\
\hline 4 & 6 & 0 & -.787524 & .747891 & .000180 \\
\hline 5 & 6 & 0 & -.191709 & -.561914 & -.000018 \\
\hline 6 & 6 & 0 & 1.270281 & -.628446 & .000294 \\
\hline 7 & 7 & 0 & -1.127334 & -1.502371 & -.000184 \\
\hline 8 & 6 & 0 & -2.311650 & -.843334 & -.000200 \\
\hline 9 & 7 & 0 & -2.127695 & .532240 & .000024 \\
\hline 10 & 7 & 0 & 1.889402 & -1.837421 & -.000375 \\
\hline 11 & 1 & 0 & 1.984414 & 2.605298 & -.000317 \\
\hline 12 & 1 & 0 & -3.290285 & -1.299920 & .000012 \\
\hline 13 & 1 & 0 & -2.865804 & 1.226502 & .000280 \\
\hline 14 & 1 & 0 & 2.901236 & -1.881408 & .002053 \\
\hline 15 & 1 & 0 & 1.355012 & -2.696576 & .001685 \\
\hline 16 & 1 & 0 & -.442811 & 2.788460 & .001304 \\
\hline \multicolumn{6}{|c|}{ - - - - - - - - - - - - - - - - - - - - - - - - - - - - - - - - - - - - - - - - - - - - - - - - - - - - - - - - - - - - - - - - - } \\
\hline Rotati & constar & $(\mathrm{GHZ}):$ & 70809 & 5067247 & .908323 \\
\hline
\end{tabular}

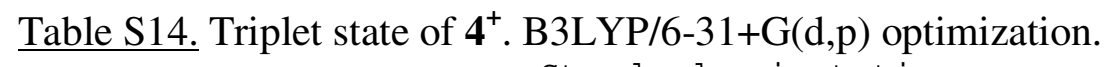

Standard orientation:

\begin{tabular}{|c|c|c|c|c|c|}
\hline \multirow{2}{*}{$\begin{array}{l}\text { Center } \\
\text { Number }\end{array}$} & \multirow{2}{*}{$\begin{array}{l}\text { Atomic } \\
\text { Number }\end{array}$} & \multirow{2}{*}{$\begin{array}{l}\text { Atomic } \\
\text { Type }\end{array}$} & \multicolumn{3}{|c|}{ Coordinates (Angstroms) } \\
\hline & & & $\mathrm{X}$ & $\mathrm{Y}$ & $\mathrm{Z}$ \\
\hline \multicolumn{6}{|c|}{ - - - - - - - - - - - - - - - - - - - - - - - - - - - - - - - - - - - - - - - - - - - - - - - - - - - - - - - } \\
\hline 1 & 7 & 0 & 1.996732 & 0.552003 & -0.008848 \\
\hline 2 & 6 & 0 & 1.362441 & 1.692484 & -0.005798 \\
\hline 3 & 7 & 0 & -0.013021 & 1.899988 & -0.001081 \\
\hline 4 & 6 & 0 & -0.715821 & 0.801031 & 0.013149 \\
\hline 5 & 6 & 0 & -0.156444 & -0.532202 & -0.002178 \\
\hline 6 & 6 & 0 & 1.263750 & -0.608958 & 0.005489 \\
\hline 7 & 7 & 0 & -1.186827 & -1.403310 & -0.016724 \\
\hline 8 & 6 & 0 & -2.448803 & -0.677795 & -0.078508 \\
\hline 9 & 7 & 0 & -2.072861 & 0.661711 & 0.017665 \\
\hline 10 & 7 & 0 & 1.967477 & -1.750441 & 0.019382 \\
\hline 11 & 1 & 0 & 1.961250 & 2.597862 & -0.012895 \\
\hline 12 & 1 & 0 & -3.307350 & -1.061969 & 0.463672 \\
\hline 13 & 1 & 0 & -2.719521 & 1.440348 & -0.004947 \\
\hline 14 & 1 & 0 & 2.980770 & -1.676811 & 0.022812 \\
\hline 15 & 1 & 0 & 1.553776 & -2.671232 & 0.076072 \\
\hline 16 & 1 & 0 & -1.140162 & -2.395201 & -0.210396 \\
\hline \multicolumn{6}{|c|}{ - - - - - - - - - - - - - - - - - - - - - - - - - - - - - - - - - - - - - - - - - - - - - - - - - - - - - - - - - - - - - - - } \\
\hline Rotatic & constar & (GHZ): & 4189 & 4640197 & 0.9066342 \\
\hline
\end{tabular}


Table S14. 6-Imino-1,6-dihydro-(1H)purine cation-radical $\left(\mathbf{6}^{+\bullet}\right)$. B3LYP/6-31+G(d,p) optimization.

\begin{tabular}{|c|c|c|c|c|c|}
\hline \multirow{2}{*}{$\begin{array}{l}\text { Center } \\
\text { Number }\end{array}$} & \multirow{2}{*}{$\begin{array}{l}\text { Atomic } \\
\text { Number }\end{array}$} & \multirow{2}{*}{$\begin{array}{l}\text { Atomic } \\
\text { Type }\end{array}$} & \multicolumn{3}{|c|}{ Coordinates (Angstroms) } \\
\hline & & & $\mathrm{X}$ & $\mathrm{Y}$ & $\mathrm{z}$ \\
\hline 1 & 7 & 0 & -1.923049 & -0.377763 & -0.000149 \\
\hline 2 & 6 & 0 & -1.397637 & -1.612650 & -0.000059 \\
\hline 3 & 7 & 0 & -0.090852 & -1.877487 & -0.000115 \\
\hline 4 & 6 & 0 & 0.673029 & -0.793647 & -0.000053 \\
\hline 5 & 6 & 0 & 0.232534 & 0.573128 & -0.000076 \\
\hline 6 & 6 & 0 & -1.177058 & 0.819716 & 0.000096 \\
\hline 7 & 7 & 0 & 1.279097 & 1.423732 & -0.000355 \\
\hline 8 & 6 & 0 & 2.347237 & 0.633221 & -0.000219 \\
\hline 9 & 7 & 0 & 2.035748 & -0.706752 & 0.000448 \\
\hline 10 & 7 & 0 & -1.852821 & 1.929664 & 0.000255 \\
\hline 11 & 1 & 0 & -2.096906 & -2.442849 & -0.000256 \\
\hline 12 & 1 & 0 & 3.371593 & 0.983203 & -0.000227 \\
\hline 13 & 1 & 0 & 2.686855 & -1.485245 & 0.001086 \\
\hline 14 & 1 & 0 & -1.231359 & 2.744633 & 0.000527 \\
\hline 15 & 1 & 0 & -2.935687 & -0.258109 & 0.000148 \\
\hline otation & constant & & 17316 & .5474606 & $0.939560 \varepsilon$ \\
\hline
\end{tabular}

Table S15. 6-Imino-3,6-dihydro-(3H)purine cation-radical $\left(7^{+*}\right)$. B3LYP/6-31+G(d,p) optimization.

\begin{tabular}{|c|c|c|c|c|c|}
\hline \multirow{2}{*}{$\begin{array}{l}\text { Center } \\
\text { Number }\end{array}$} & \multirow{2}{*}{$\begin{array}{l}\text { Atomic } \\
\text { Number }\end{array}$} & \multirow{2}{*}{$\begin{array}{l}\text { Atomic } \\
\text { Type }\end{array}$} & \multicolumn{3}{|c|}{ Coordinates (Angstroms) } \\
\hline & & & $\mathrm{x}$ & $\mathrm{Y}$ & z \\
\hline 1 & 7 & 0 & 2.028461 & 0.322254 & -0.000009 \\
\hline 2 & 6 & 0 & 1.534540 & 1.513815 & 0.000189 \\
\hline 3 & 7 & 0 & 0.168163 & 1.795003 & 0.000245 \\
\hline 4 & 6 & 0 & -0.684027 & 0.756262 & 0.000146 \\
\hline 5 & 6 & 0 & -0.209552 & -0.590157 & -0.000049 \\
\hline 6 & 6 & 0 & 1.207298 & -0.804337 & -0.000153 \\
\hline 7 & 7 & 0 & -1.265961 & -1.448192 & -0.000228 \\
\hline 8 & 6 & 0 & -2.332371 & -0.685348 & 0.000190 \\
\hline 9 & 7 & 0 & -2.034600 & 0.681200 & 0.000087 \\
\hline 10 & 7 & 0 & 1.703961 & -2.024898 & -0.000446 \\
\hline 11 & 1 & 0 & 2.182568 & 2.385373 & 0.000286 \\
\hline 12 & 1 & 0 & -3.357657 & -1.032237 & 0.000181 \\
\hline 13 & 1 & 0 & -2.708543 & 1.438432 & 0.000274 \\
\hline 14 & 1 & 0 & 2.730720 & -1.974116 & -0.000498 \\
\hline 15 & 1 & 0 & -0.142577 & 2.763564 & 0.000281 \\
\hline $\begin{array}{l}----- \\
\text { Rotat }\end{array}$ & nat & 7) & $\begin{array}{l}---\cdots-1 \\
3814828\end{array}$ & - & $0.936665]$ \\
\hline
\end{tabular}


Table S16. 6-Imino-6,7-dihydro-(7H)purine cation-radical $\left(\mathbf{8}^{+\bullet}\right)$. B3LYP/6-31+G(d,p) optimization.

\begin{tabular}{|c|c|c|c|c|c|}
\hline \multirow{2}{*}{$\begin{array}{l}\text { Center } \\
\text { Number }\end{array}$} & \multirow{2}{*}{$\begin{array}{l}\text { Atomic } \\
\text { Number }\end{array}$} & \multirow{2}{*}{$\begin{array}{c}\text { Atomic } \\
\text { Type }\end{array}$} & \multicolumn{3}{|c|}{ Coordinates (Angstroms) } \\
\hline & & & $\mathrm{x}$ & $\mathrm{Y}$ & Z \\
\hline 7 & 7 & 0 & $x^{2}-1050700$ & - & - \\
\hline $\begin{array}{l}1 \\
2\end{array}$ & 6 & 0 & $\begin{array}{l}-2.050129 \\
-1.509154\end{array}$ & $\begin{array}{r}-.346841 \\
-1.564156\end{array}$ & $\begin{array}{r}.000043 \\
-.000035\end{array}$ \\
\hline 3 & 7 & 0 & -.188529 & -1.880732 & .000029 \\
\hline 4 & 6 & 0 & .592695 & -.813315 & .000025 \\
\hline 5 & 6 & 0 & .158733 & .512456 & .000006 \\
\hline 6 & 6 & 0 & -1.245782 & .757543 & .000000 \\
\hline 7 & 7 & 0 & 1.287813 & 1.307218 & -.000015 \\
\hline 8 & 6 & 0 & 2.372223 & .520312 & .000167 \\
\hline 9 & 7 & 0 & 1.988539 & -.761603 & -.000165 \\
\hline 10 & 7 & 0 & -1.693992 & 2.009800 & -.000043 \\
\hline 11 & 1 & 0 & -2.195858 & -2.404733 & .000285 \\
\hline 12 & 1 & 0 & 3.395820 & .868077 & .000342 \\
\hline 13 & 1 & 0 & 2.610260 & -1.565263 & -.000325 \\
\hline 14 & 1 & 0 & -2.721356 & 2.006238 & -.000100 \\
\hline 15 & 1 & 0 & 1.292932 & 2.323753 & -.000137 \\
\hline Rotatic & const & ( GH: & 4188626 & 1.5379161 & .9401 \\
\hline
\end{tabular}

Table S17. 6-Amino-1,2-dihydro-(1H)purine-2-yl cation-radical $\left(\mathbf{9}^{+*}\right)$. B3LYP/6-31+G(d,p) optimization.

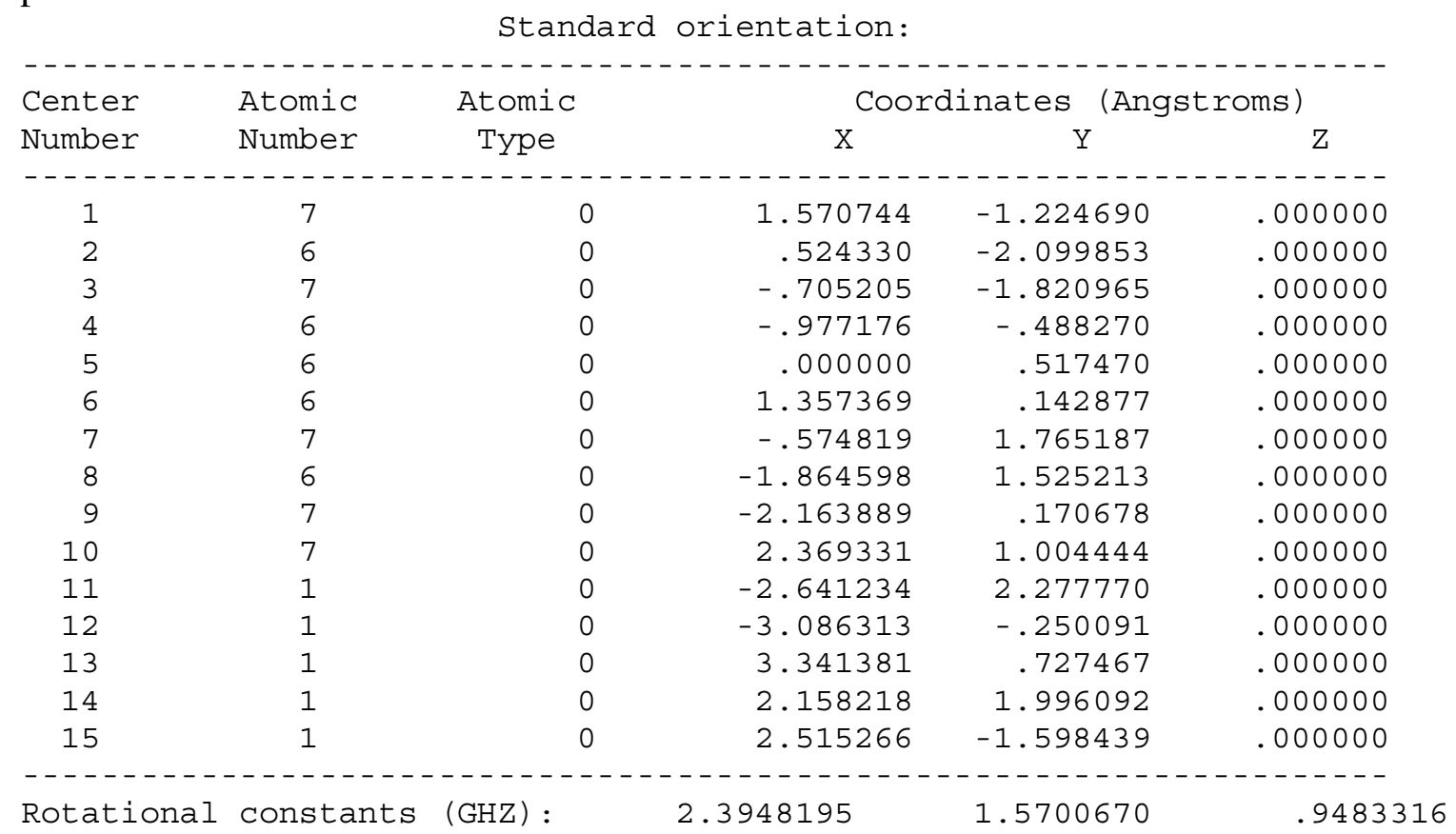


Table S18. 6-Amino-1,2-dihydro-(1H)purine-8-yl cation-radical (10 $\left.{ }^{+*}\right)$. B3LYP/6-31+G(d,p) optimization.

\begin{tabular}{|c|c|c|c|c|c|}
\hline \multicolumn{6}{|c|}{ Standard orientation: } \\
\hline Center & Atomic & Atomic & \multicolumn{3}{|c|}{ Coordinates (Angstroms) } \\
\hline Number & Number & Type & $\mathrm{X}$ & $\mathrm{Y}$ & Z \\
\hline \multicolumn{6}{|c|}{ - - - - - - - - - - - - - - - - - - - - - - - - - - - - - - - - - - - - - - - - - - - - - - - - - - - - - - - - - - } \\
\hline 1 & 7 & 0 & 1.777834 & .624792 & .000000 \\
\hline 2 & 6 & 0 & 1.074662 & 1.815964 & .000000 \\
\hline 3 & 7 & 0 & -.223693 & 1.897446 & .000000 \\
\hline 4 & 6 & 0 & -.838945 & .699303 & .000000 \\
\hline 5 & 6 & 0 & -.221660 & -.561504 & .000000 \\
\hline 6 & 6 & 0 & 1.181379 & -.612384 & .000000 \\
\hline 7 & 7 & 0 & -1.167781 & -1.573067 & .000000 \\
\hline 8 & 6 & 0 & -2.280263 & -.941220 & .000000 \\
\hline 9 & 7 & 0 & -2.187070 & .427366 & .000000 \\
\hline 10 & 7 & 0 & 1.896138 & -1.736505 & .000000 \\
\hline 11 & 1 & 0 & 1.683533 & 2.713775 & .000000 \\
\hline 12 & 1 & 0 & -2.946877 & 1.098076 & .000000 \\
\hline 13 & 1 & 0 & 2.906359 & -1.757015 & .000000 \\
\hline 14 & 1 & 0 & 1.406428 & -2.623289 & .000000 \\
\hline 15 & 1 & 0 & 2.791524 & .687272 & .000000 \\
\hline \multicolumn{6}{|c|}{$\begin{array}{lll}\text { Rotational constants (GHZ): } & 2.3128132 & 1.6122672\end{array}$} \\
\hline Rotatio & constan & (GHZ): & 3128132 & 1.6122672 & .9500118 \\
\hline
\end{tabular}

Table S19. 6-Amino-2,3-dihydro-(3H)purine-2-yl cation-radical (11+*). B3LYP/6-31+G(d,p) optimization.

\begin{tabular}{|c|c|c|c|c|c|}
\hline \multicolumn{6}{|c|}{ Standard orientation: } \\
\hline----- & ------- & ------- & ------ & ---------- & -------- \\
\hline \multirow{2}{*}{$\begin{array}{l}\text { Center } \\
\text { Number }\end{array}$} & \multirow{2}{*}{$\begin{array}{l}\text { Atomic } \\
\text { Number }\end{array}$} & \multirow{2}{*}{$\begin{array}{l}\text { Atomic } \\
\text { Type }\end{array}$} & \multicolumn{3}{|c|}{ Coordinates (Angstroms) } \\
\hline & & & $\mathrm{X}$ & $\mathrm{Y}$ & $\mathrm{Z}$ \\
\hline \multicolumn{6}{|c|}{ 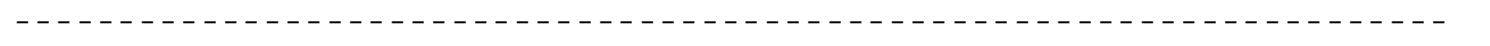 } \\
\hline 1 & 7 & 0 & 1.715606 & -1.102053 & 0.000000 \\
\hline 2 & 6 & 0 & 0.832510 & -2.002283 & 0.000000 \\
\hline 3 & 7 & 0 & -0.508381 & -1.837851 & 0.000000 \\
\hline 4 & 6 & 0 & -0.916657 & -0.523300 & 0.000000 \\
\hline 5 & 6 & 0 & 0.000000 & 0.529258 & 0.000000 \\
\hline 6 & 6 & 0 & 1.390069 & 0.231608 & 0.000000 \\
\hline 7 & 7 & 0 & -0.658917 & 1.737449 & 0.000000 \\
\hline 8 & 6 & 0 & -1.927763 & 1.428644 & 0.000000 \\
\hline 9 & 7 & 0 & -2.146760 & 0.050603 & 0.000000 \\
\hline 10 & 7 & 0 & 2.346863 & 1.143225 & 0.000000 \\
\hline 11 & 1 & 0 & -2.753631 & 2.126664 & 0.000000 \\
\hline 12 & 1 & 0 & -3.050076 & -0.407550 & 0.000000 \\
\hline 13 & 1 & 0 & 3.319629 & 0.862128 & 0.000000 \\
\hline 14 & 1 & 0 & 2.114698 & 2.129055 & 0.000000 \\
\hline 15 & 1 & 0 & -1.138449 & -2.633473 & 0.000000 \\
\hline \multicolumn{6}{|c|}{ - - - - - - - - - - - - - - - - - - - - - - - - - - - - - - - - - - - - - - - - - - - - - - - - - - - - - - - - - - - - - - - - - - - - - } \\
\hline Rotatic & constan & (GHZ) & 3811023 & 1.5683344 & 0.94554 \\
\hline
\end{tabular}


Table S20. 6-Amino-2,3-dihydro-(3H)purine-8-yl cation-radical (12+*). B3LYP/6-31+G(d,p) optimization.

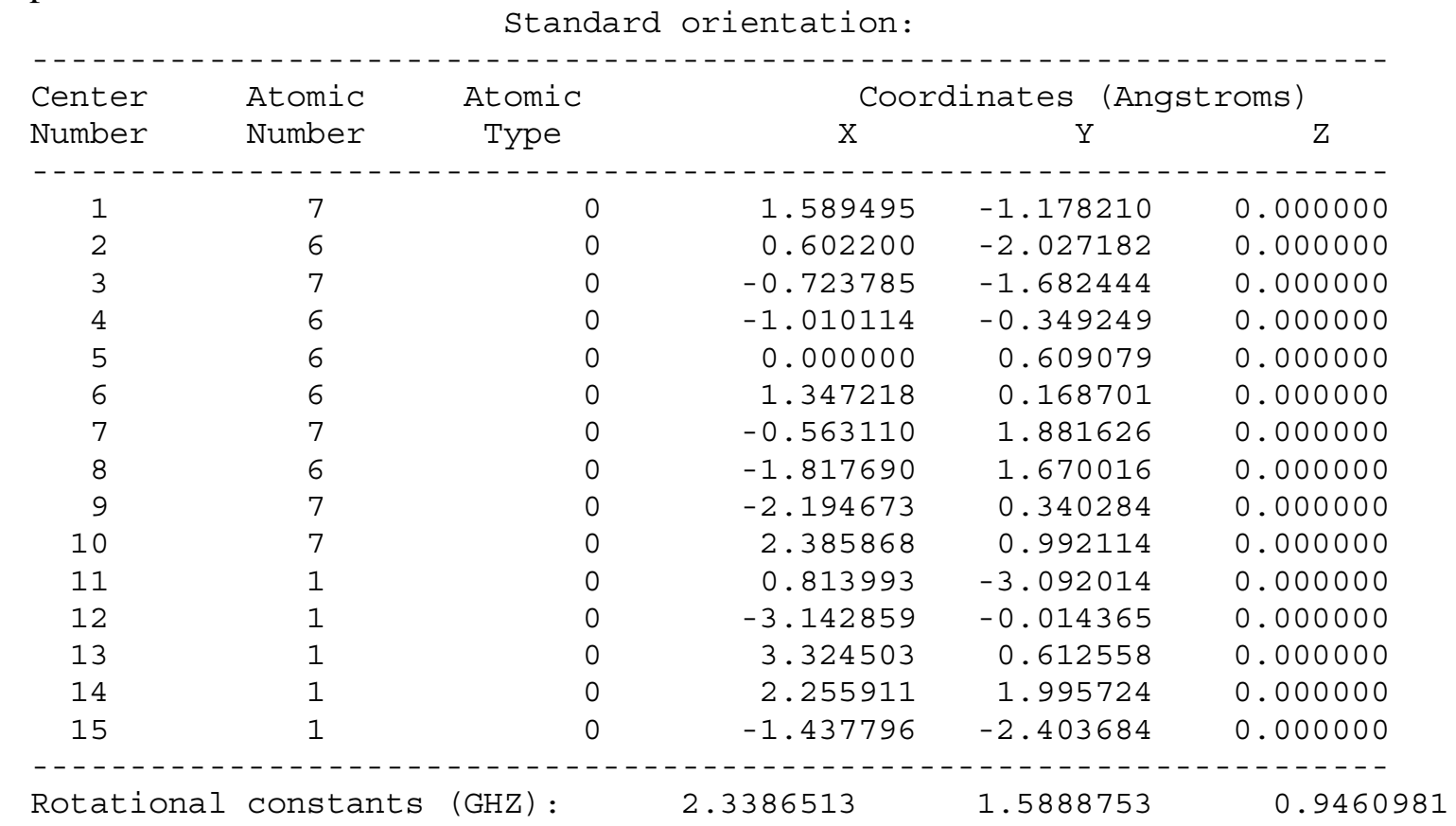

Table S21. 6-Amino-7,8-dihydro-(7H)purine-2-yl cation-radical (13+*). B3LYP/6-31+G(d,p) optimization.

\begin{tabular}{|c|c|c|c|c|c|}
\hline \multirow{2}{*}{$\begin{array}{l}\text { Center } \\
\text { Number }\end{array}$} & \multirow{2}{*}{$\begin{array}{l}\text { Atomic } \\
\text { Number }\end{array}$} & \multirow{2}{*}{$\begin{array}{l}\text { Atomic } \\
\text { Type }\end{array}$} & \multicolumn{3}{|c|}{ Coordinates (Angstroms) } \\
\hline & & & $\mathrm{x}$ & $\mathrm{Y}$ & $\mathrm{Z}$ \\
\hline & & & ---- & - - - & ------- \\
\hline 1 & 7 & 0 & -1.929283 & .749019 & .000000 \\
\hline 2 & 6 & 0 & -1.173960 & 1.812424 & .000000 \\
\hline 3 & 7 & 0 & .133292 & 1.966059 & .000000 \\
\hline 4 & 6 & 0 & .719526 & .771308 & .000000 \\
\hline 5 & 6 & 0 & .073561 & -.471748 & .000000 \\
\hline 6 & 6 & 0 & -1.351580 & -.470788 & .000000 \\
\hline 7 & 7 & 0 & 1.086658 & -1.434596 & .000000 \\
\hline 8 & 6 & 0 & 2.274597 & -.824220 & .000000 \\
\hline 9 & 7 & 0 & 2.086181 & .500285 & .000000 \\
\hline 10 & 7 & 0 & -2.140290 & -1.555898 & .000000 \\
\hline 11 & 1 & 0 & 3.232520 & -1.324145 & .000000 \\
\hline 12 & 1 & 0 & 2.817807 & 1.204692 & .000000 \\
\hline 13 & 1 & 0 & -3.142373 & -1.410869 & .000001 \\
\hline 14 & 1 & 0 & -1.798219 & -2.503466 & .000003 \\
\hline 15 & 1 & 0 & .981488 & -2.442168 & -.000001 \\
\hline
\end{tabular}


Table S22. 6-Amino-7,8-dihydro-(7H)purine-8-yl cation-radical (14+*). B3LYP/6-31+G(d,p) optimization.

\begin{tabular}{|c|c|c|c|c|c|}
\hline \multirow{2}{*}{$\begin{array}{l}\text { Center } \\
\text { Number }\end{array}$} & \multirow{2}{*}{$\begin{array}{l}\text { Atomic } \\
\text { Number }\end{array}$} & \multirow{2}{*}{$\begin{array}{l}\text { Atomic } \\
\text { Type }\end{array}$} & \multicolumn{3}{|c|}{ Coordinates (Angstroms) } \\
\hline & & & $\mathrm{x}$ & $\mathrm{Y}$ & $\mathrm{Z}$ \\
\hline 1 & 7 & 0 & 1.894502 & .634228 & .020658 \\
\hline 2 & 6 & 0 & 1.190625 & 1.760523 & .012190 \\
\hline 3 & 7 & 0 & -.161207 & 1.890927 & -.022182 \\
\hline 4 & 6 & 0 & -.775375 & .734462 & -.058804 \\
\hline 5 & 6 & 0 & -.164741 & -.541115 & -.070972 \\
\hline 6 & 6 & 0 & 1.261952 & -.555800 & -.000326 \\
\hline 7 & 7 & 0 & -1.198281 & -1.462349 & -.053670 \\
\hline 8 & 6 & 0 & -2.379786 & -.820439 & .112879 \\
\hline 9 & 7 & 0 & -2.153666 & .492095 & .011461 \\
\hline 10 & 7 & 0 & 2.015967 & -1.662047 & .030527 \\
\hline 11 & 1 & 0 & 1.757994 & 2.685640 & .036600 \\
\hline 12 & 1 & 0 & -2.860155 & 1.211987 & .129480 \\
\hline 13 & 1 & 0 & 3.022918 & -1.545055 & .072074 \\
\hline 14 & 1 & 0 & 1.642970 & -2.600042 & .047616 \\
\hline 15 & 1 & 0 & -1.140985 & -2.468296 & -.163133 \\
\hline----- & & - & $\begin{array}{l}---\cdots- \\
961184\end{array}$ & 5344837 & 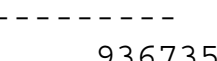 \\
\hline
\end{tabular}



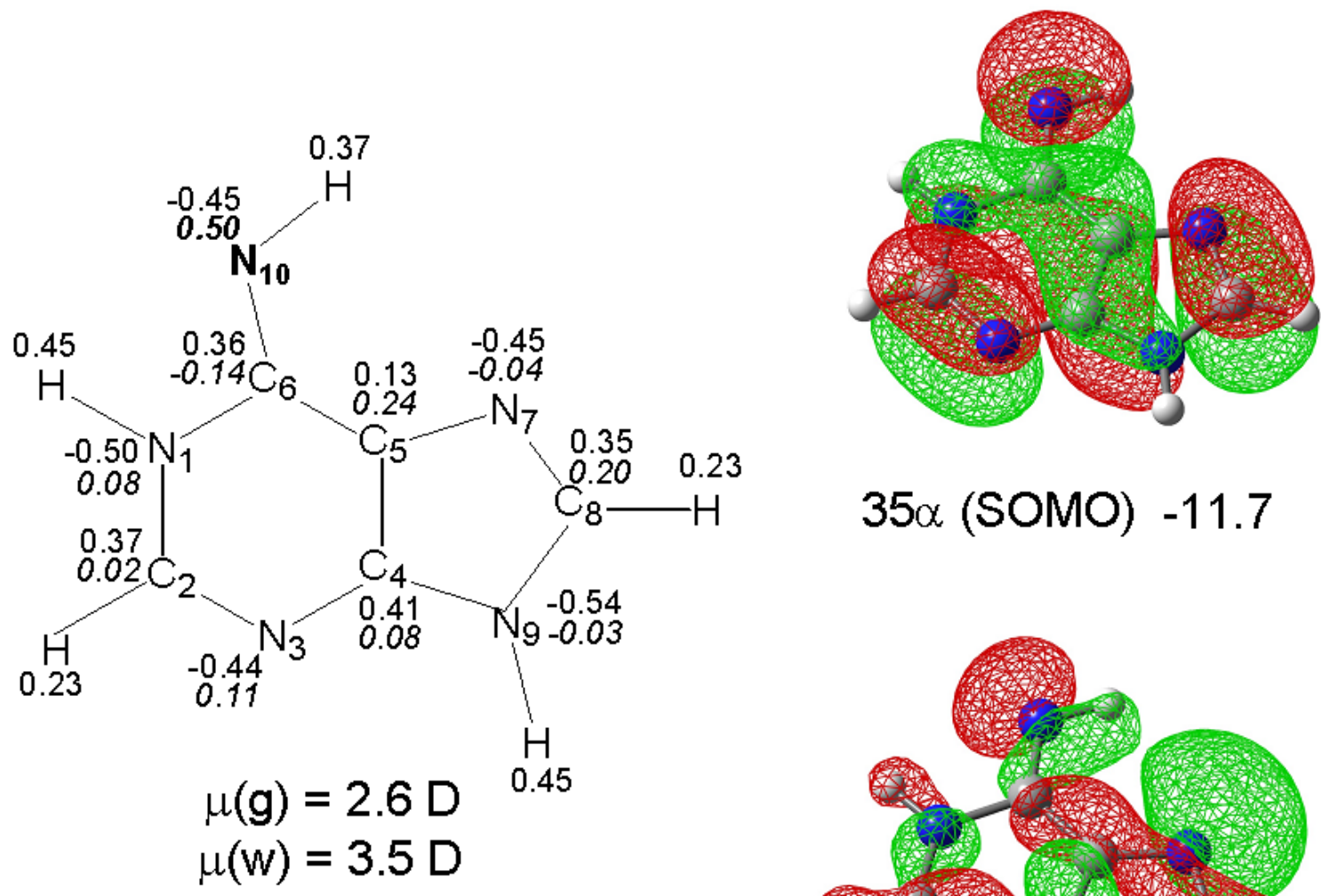

$35 \alpha$ (SOMO) -11.7

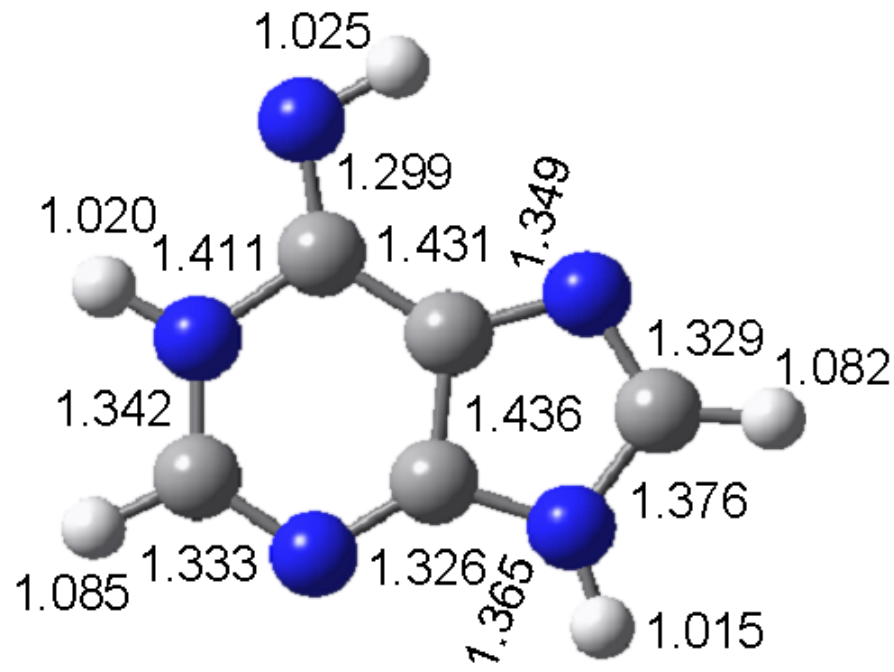

\section{$6^{+\bullet}$}

Figure 6
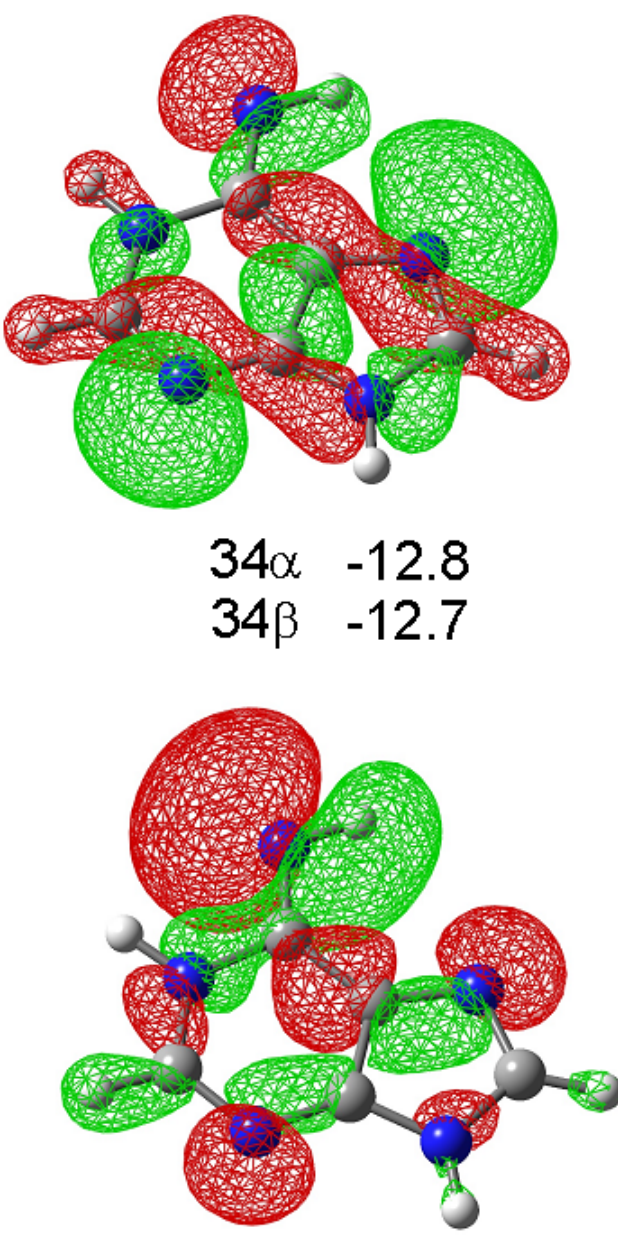

$33 \alpha-13.4$

$33 \beta \varepsilon=-13.0 \mathrm{eV}$

Figure S1. B3LYP/6-31+G(d,p) optimized structure of $\mathbf{6}^{+\bullet}$. Description as in Figure 5. 


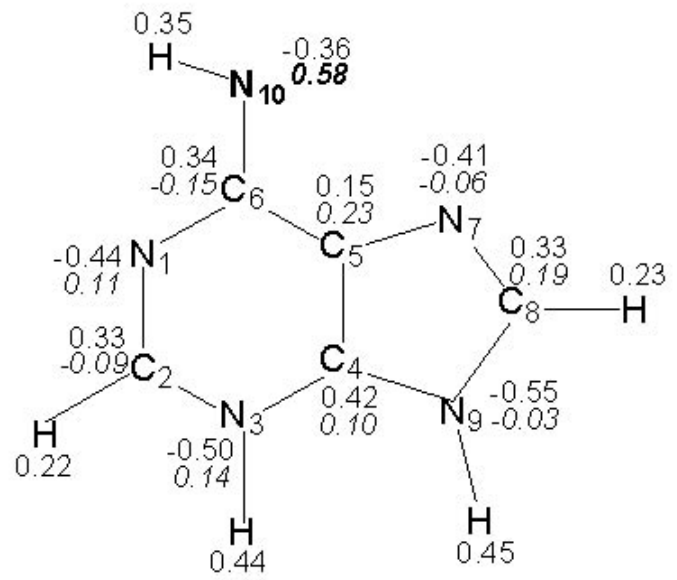

$$
\begin{aligned}
& \mu(\mathrm{g})=8.3 \mathrm{D} \\
& \mu(\mathrm{w})=12.3 \mathrm{D}
\end{aligned}
$$

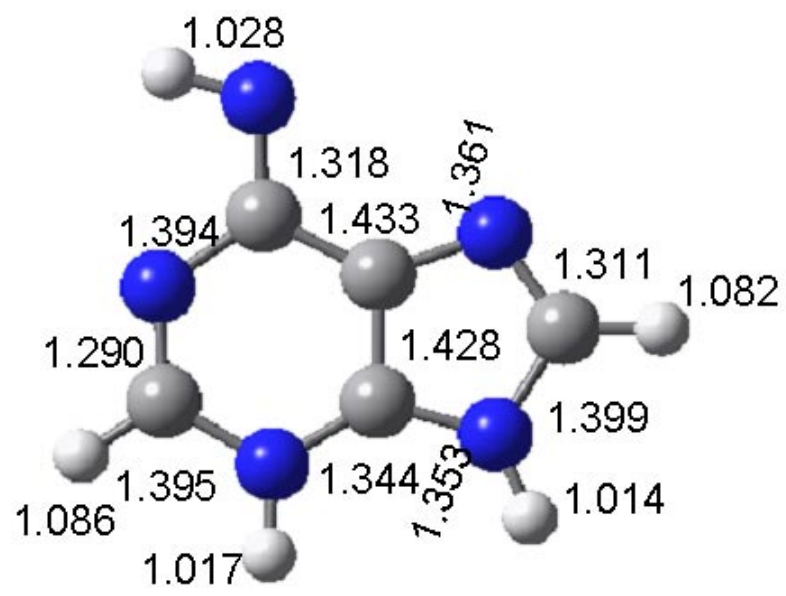

$7^{+\infty}$

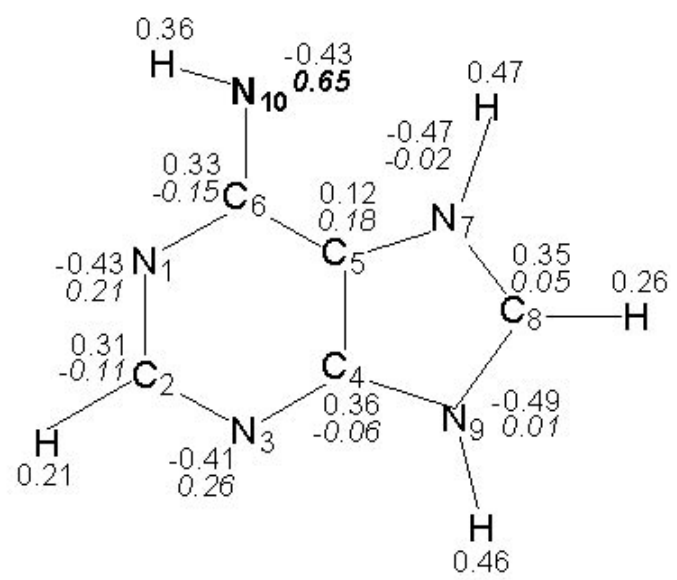

$\mu(\mathrm{g})=6.8 \mathrm{D}$ $\mu(w)=9.8 D$

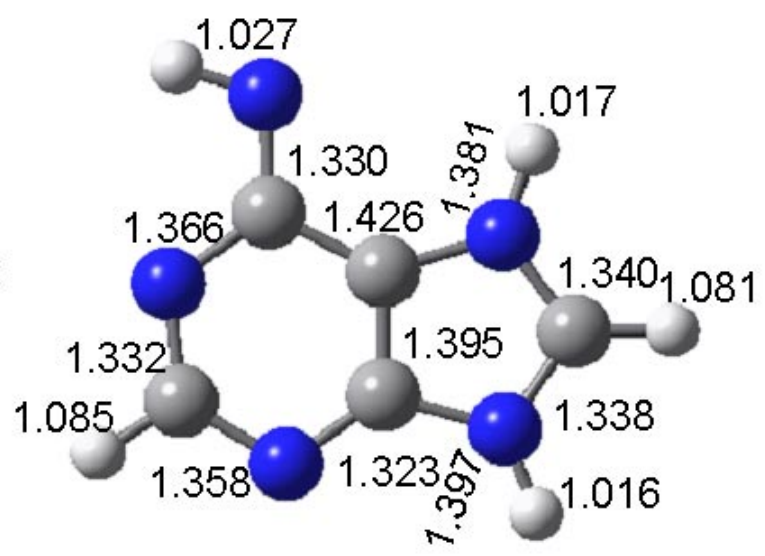

$8^{+\bullet}$

Figure S2. $\quad$ B3LYP/6-31+G(d,p) optimized structures of $\mathbf{7}^{+\bullet}$ and $\mathbf{8}^{+\bullet}$. 

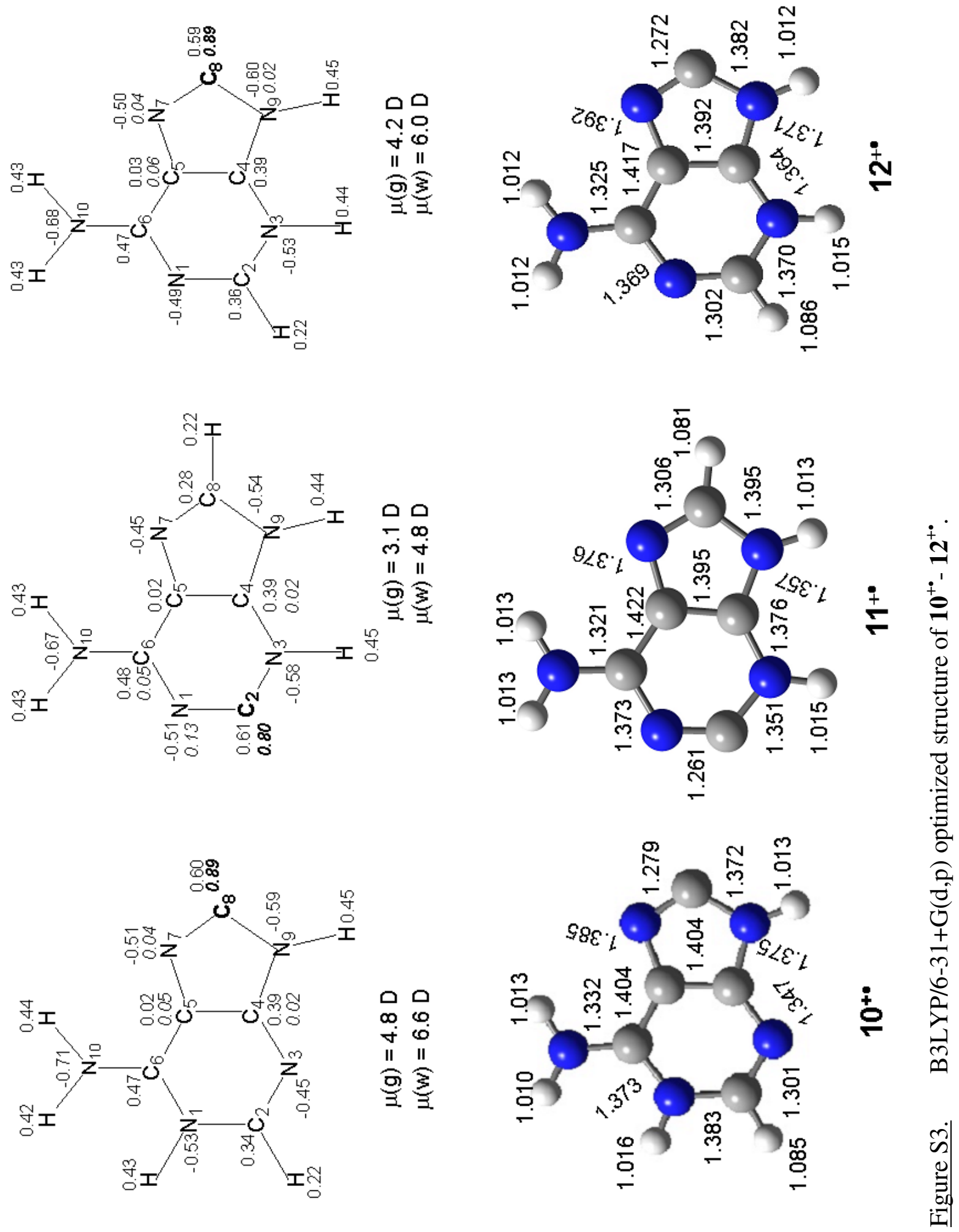

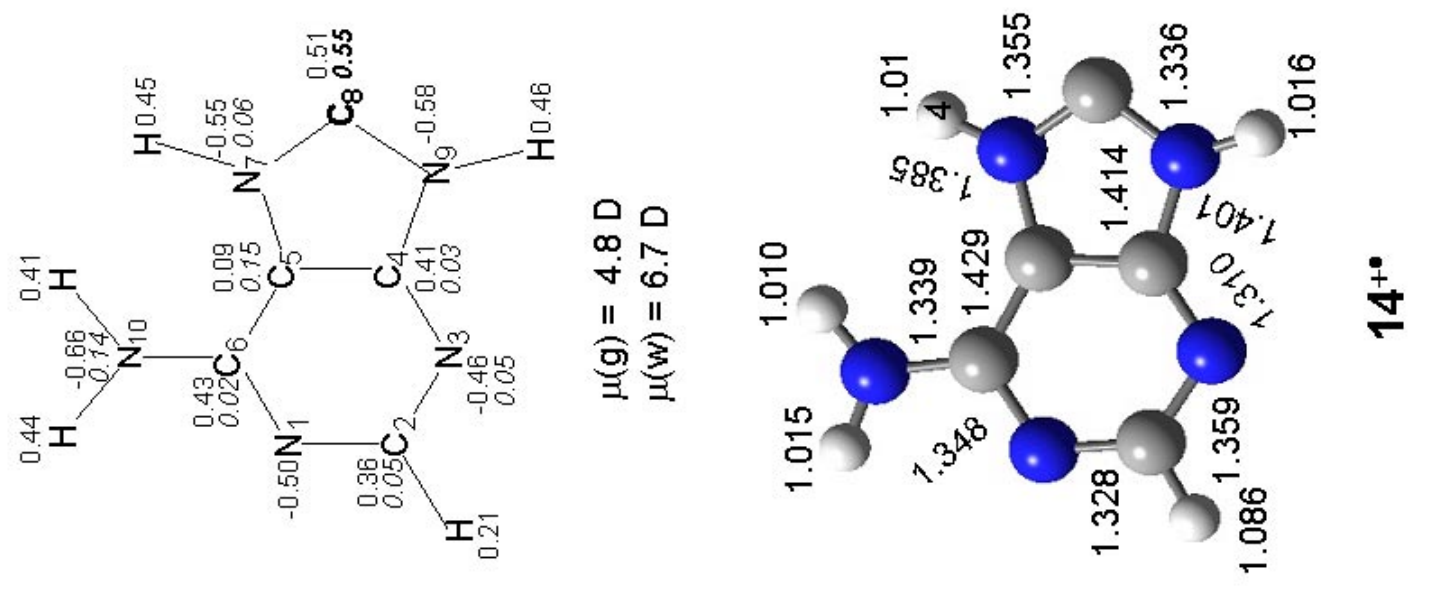

艺
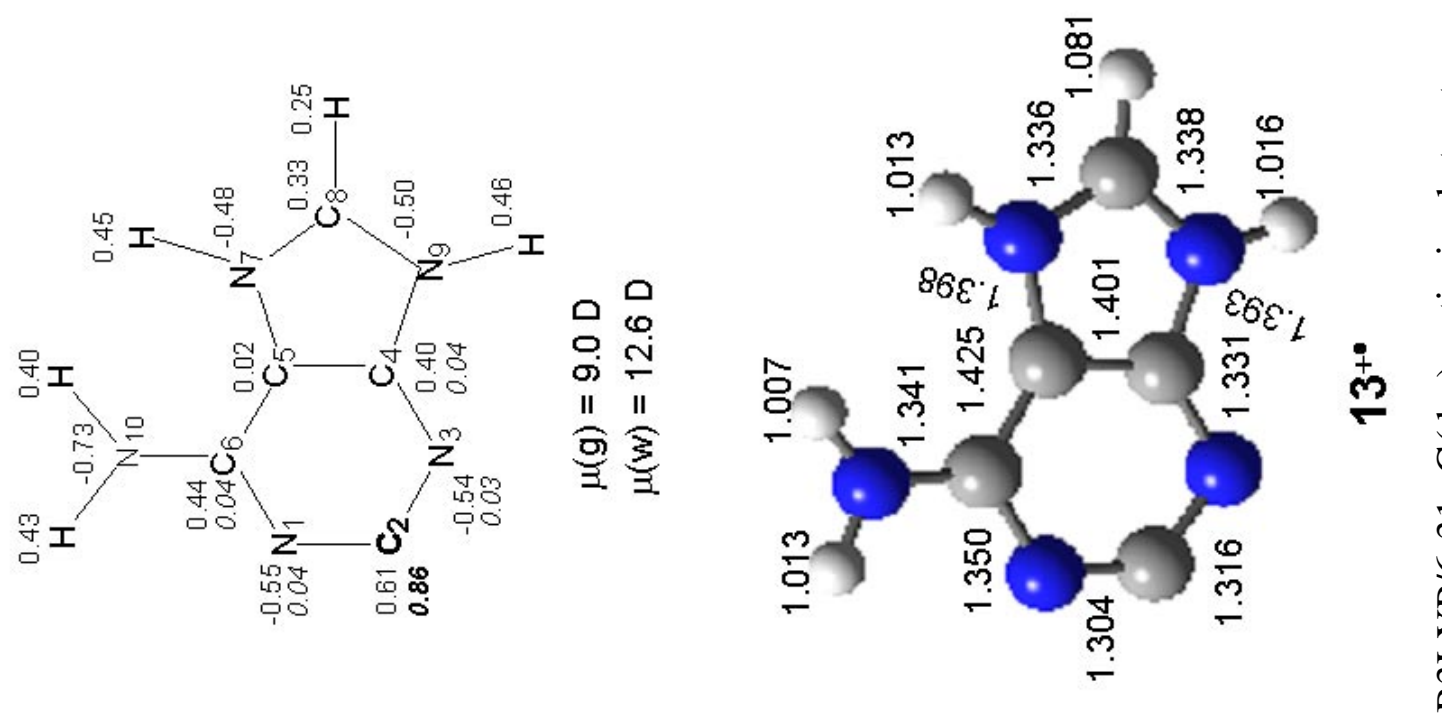

$\stackrel{+}{m}$



름

용

ํㅡㅇ

2)

芒

苟 